\title{
Categorization of Green and Grey Infrastructure Complexity in the Rural-Urban Interface of Bengaluru, India; an unsupervised volumetric approach with relevance for urban quality
}

Nils Nölke ( $\sim$ nnoelke@gwdg.de)

University of Göttingen: Georg-August-Universitat Gottingen https://orcid.org/0000-0003-4925-2287 Lutz Fehrmann University of Göttingen: Georg-August-Universitat Gottingen

\section{Tobias Plieninger}

University of Göttingen: Georg-August-Universitat Gottingen

\section{Christoph Kleinn}

University of Göttingen: Georg-August-Universitat Gottingen

\section{Research Article}

Keywords: Rurban configurations, Satellite imagery, Machine learning, 3D metric

Posted Date: January 19th, 2022

DOI: https://doi.org/10.21203/rs.3.rs-1155273/v1

License: (c) (1) This work is licensed under a Creative Commons Attribution 4.0 International License.

Read Full License 


\section{Abstract}

Trees are key elements of urban green infrastructure and provide multiple ecosystem services that are essential for the quality of life of people in an urban environment. Grey infrastructure is made up of buildings or built-up area, generally characterized by imperviousness of the surface. The complexity of urban green and grey infrastructure and their interactions co-define the quality of urban life and the ecological value. Using conventional dichotomies by separation into „urban" and „rural" contexts does hardly allow to comprehensively assess the situation in rapidly and often chaotically urbanizing environments of the Global South. We present an unsupervised remote sensing-based approach integrating 3D information as an approach to objectively categorize the complexity of green and grey infrastructure. For our study area in Bengaluru, India, we distinguished five categories describing composition and configuration of the green and grey infrastructure, where three variables served as indicators for categorization into five cluster. We argue that such integrated 3D assessment of green and grey infrastructure is particularly useful for understanding and classifying "rurban" environments, where a distinction between urban and rural is often no longer possible The final map allows to quantitatively characterize rurban configurations.

\section{Introduction}

The Earth's land surface is undergoing rapid and manifold changes. Among the most drastic land use changes is the sealing of land surface by infrastructure or building constructions, generating so-called „impervious surfaces“ (Seto et al., 2011). Such areas are core elements of builtup areas, settlements, villages, and urban areas. Estimated at about $0.45 \%$ of the habitable land area in 2010 (Liu et al., 2014), the global total of settlement and urban area is relatively small in comparison to agricultural land cover (about $50 \%$ ) and forests (about $35 \%$ ), but urban areas are constantly and rapidly expanding. A welldefined and unambiguous definition is therefore a must when reliable land use maps shall be produced, also due to the substantial impacts on sustainability of urban areas (Elmqvist et al., 2021). There is a multitude of possible definitions for the degree of urbanization: the Statistical Office of the European Union (Eurostat) uses, for example, a criterion of geographical contiguity in combination with a minimum population threshold within $1 \mathrm{~km}^{2}$ square grid cells (European Commission. Statistical Office of the European Union., 2021). For remote sensing image interpretation, however, one needs in the first place to resort to observable biophysical features, since social and economic variables cannot be identified directly from the imagery. The most typical biophysical feature of settlements and urban areas is the presence of built-up elements, including houses, industrial areas, roads, and parking lots. In a hierarchical classification framework, it appears straightfoward to start out with an identification of impervious surfaces and proceed with a classification into classes characterizing the degree of urbanity, either in categories (such as "urban", "transition", "rural") or on a continuous scale of, for example, percent of "urbanity" on the second level.

Research on quantification of urban impervious surface and its spatial pattern is commonly based on remote sensing analysis (Elvidge et al., 2007, Ma, 2016). Yet mapping the class "urban" is the central 
element in urban studies, and research has considered various spatial resolutions as well as different sensors and classification methods (Weng, 2012). High-resolution satellite imagery such as IKONOS, QuickBird or WorldView are the premier data source for detailed thematic mapping of urbanity (Goetz et al., 2003; Hu and Weng, 2011; Cablk and Minor, 2003). Impervious surface is used as proxy variable as the class "urban" cannot be directly classified from remote sensing

Within the assessment of urbanization, urban green spaces play an important role. Urban green spaces, also referred to as green infrastructure (Hansen et al., 2019), are composed of parks, gardens, roadside trees or alleys, and other "green" landscape elements and have a crucial importance for regulating air temperature (Herath et al., 2018) and air quality (Nowak et al., 2006) and also for providing habitats to animals and plants (Lepczyk et al., 2017). Various studies have shown that the occurrence of green spaces in the neighbourhood has a positive effect on health and well-being (Groenewegen, 2006; Maas et al., 2009; Troy and Grove, 2008), in particular where trees form the main element of green infrastructure.

Conventional methods for assessing and analysing urbanization and its complexity are usually based on gradient analysis or on the use of concentric circles but may fail to assess a new type of environment, which is found around many of the rapidly urbanising megacities of the Global South.. Such environments are characterised by both urban and rural features. For example, despite intensive development of traffic and settlement infrastructure, they are often characterised by a high presence of green spaces which could be either historical or newly created. The megacity of Bangalore in the South of India is one example of such new environment, with e.g. old-growth tree structures overrun by urbanization, so that historic green spaces or remnants of agricultural use are frequently found next to new and very dense settlements. An enormous complexity of grey and green infrastructure emerges here, both in terms of (two-dimensional) area and the (three-dimensional) space-filling built-up volume.

High-resolution satellite imagery allows assessing and categorizing the complex pattern of grey and green infrastructure at the rural-urban interface through quantitative analyses. Previous studies have commonly used data derived in a two-dimensional domain in combination with hard thresholding, frequently ignoring the important fact that urbanization takes also place in a three-dimensional space. Therefore, it may be indicated to integrate the third dimension when doing a classification of urbanity. The aim of this study is to fill this gap by developing a novel, transparent, and unambiguous approach to extract quantitative and comparable 2D/3D information on the degree of urbanity and as basis for a categorization of grey and green infrastructure. The approach we propose here uses a small set of biophysical variables, extracted from high-resolution remote sensing imagery, as indicators.

\section{Materials And Methods}

\subsection{Study area}

Bengaluru is the capital of the Indian State of Karnataka, located at $12^{\circ} 58^{\prime} \mathrm{N}, 77^{\circ} 35^{\prime} \mathrm{E}$. It is situated on Southern India's Deccan plateau at an altitude of about $920 \mathrm{~m}$ above MSL (Sudhira and Nagendra, 2013). 
The topography is relatively flat in Bengaluru North, which is one of four urban districts, while Bengaluru South is slightly undulating, with a central ridge running in North-East and South-West direction. The city is nowadays a centre of IT, biotechnology, aerospace technology, and other advanced knowledge-based industries and research centers (Hiremath et al., 2013). Bengaluru used to be known as the 'garden city' of India owing to its widespread parks, green spaces and many alleys with old and huge trees. Even though many of these natural areas and elements have been lost due to infrastructure development, trees and green spaces remain abundant as compared to other megacities (Nagendra, 2016).

Our analysis uses data from a $50 \mathrm{~km} \times 5 \mathrm{~km}$ rectangular research transect (Fig. 1) in the Northern part of Bengaluru that has been defined in the framework of an Indian-German collaborative research project (Hoffmann et al., 2017). This transect covers a wide range from densely built-up urban environments to areas that have a purely rural character. The unusually complex pattern of green and grey infrastructure is what makes Bengaluru an interesting and challenging study site for developing the approach presented here.

\subsection{Remote sensing imagery}

Our Bengaluru image dataset covers the transect area of $250 \mathrm{~km}^{2}$ and was acquired on 2016-11-16 by WorldView-3 (Digital Globe ${ }^{\circledR}$ ) under cloud-free conditions. WorldView-3 has eight VNIR bands with a nominal resolution of $1.24 \mathrm{~m}$ and one panchromatic band with resolution of $0.31 \mathrm{~m}$. We performed a datafusion to rise the resolution of the multi-spectral bands to $0.3 \mathrm{~m}$, based on a pan-sharpening method implemented in Geomatica Banff by PCI Geomatics (PCl Geomatics, Richmond Hill, Ontario, Canada).

\subsection{Terminology and definition}

For any classification of land cover and land use, clear definitions are crucial. A meaningful interpretation of the results can only be warranted when a comprehensive, transparent, and unambiguous definition of all elements is available and consistently used in the classification process. In our context, we use a hierarchical "three-level framework" of definitions of classes and terminology, where the first level is assigned to individual pixels and further levels include an evaluation of the neighborhood inside a defined reference area:

- 1st level - "Impervious": Impervious is a characteristic of the land surface and describes mainly artificial structures which are usually water-impermeable. Imperviousness can be determined at each (dimensionless) point; from bird's eye view either a point falls on an impervious surface or not. In order to make an accurate mapping from remote sensing, very high-resolution imagery is required if one wishes to follow this definitional element. In our case, this is the WorldView-3 imagery with a nominal ground resolution of about $0.3 \mathrm{~m}$. The identification of "Impervious" from high-resolution remote sensing imagery is quite straightforward in many cases.

- 2nd level - "Urban": Determining the status of "urban" or "rural" is more complex, since the classification is based on a mix of biophysical and socio-economic characteristics. In this study, we consider percent impervious surface (PIC), a two-dimensional biophysical characteristic exclusively. 
When assigning the mean PIC to a location (or to a single pixel), the definition of a "reference area" of different sizes around that point is required. The relevance of the definition of a reference area when dealing with percent cover has been addressed early by Kleinn (2001), and in the remote sensing context by Magdon et al. (2014).

- 3rd level - "Green and grey infrastructure complexity": While in level 2 the grey infrastructure is represented by the mean PIC in the 2D space, in the third level, the complexity is extended to the 3D space to jointly categorize the configuration and complexity of green and grey infrastructure, derived from biophysical characteristics of remote sensing imagery. The biophysical variables include building volume, tree- or green vegetation volume, and the mean impervious cover percent in the surrounding area from the 2 nd level.

For the 1 st and 2 nd level, the class is assigned to individual pixels. For "impervious surface" (level 1 ) this decision is made exclusively from information of the pixel itself: the pixel is on impervious surface or not. Given the very high spatial resolution, mixed pixels can be ignored as they occur in a relatively small proportion only. For "degree of urbanity" (level 2) a support area needs to be evaluated in the immediate surroundings of the target pixel to derive the relative proportion of impervious surface. It is then a matter of definition how large this support area shall be and which shape it should have. The 3rd level is assigned to a reference area of one hectare, a common size in urban studies (Schoepfer et al., 2005).

\subsection{U-Net classification (1st level)}

We classified impervious surface, built-up area, and tree cover for the $5 \times 50 \mathrm{~km}$ transect from the WorldView-3 image using a deep learning approach. We used a convolutional neural network called U-Net (Ronneberger et al., 2015) with a network structure very similar to the one implemented by Iglovikov et al. (2017), along with the published joint loss function. The network receives images with a size of $112 \times 112$ pixels as input and produces a probability map of $72 \times 72$ pixels as output. The implementation was done using the Keras (Chollet, 2017) framework with TensorFlow as backend (Martin Abadi et al., 2005). Training and validation were performed on random subsets of the 330 tiles covering the 1 ha sample plots. The split into training and test data was $70-30 \%$. Training was done for 100 epochs, where in each epoch 135 batches of 16 images were presented to the network. The total image area fed to the network in each epoch was equal to half of the image area of the training set. Only four (blue, green, red, nearinfrared) of the eight WorldView-3 bands were used, as it proved to be enough for this classification task (Freudenberg et al., 2019). In order to prevent over-fitting, the input images were augmented by random flipping and $90^{\circ}$ rotations.

\subsection{Mean impervious cover \% (2nd level)}

To produce a continuous map of the degree of urbanity, first a moving window approach was applied. Extracting the relative proportion of impervious surface (Percent Impervious Cover $=P I C$ ) for window sizes of $100 \mathrm{~m} \times 100 \mathrm{~m}, 400 \mathrm{~m} \times 400 \mathrm{~m}$ and $800 \mathrm{~m} \times 800 \mathrm{~m}$ allowing to describe the neighborhood on 
different spatial scales, from neighboring houses to ward or village level. The workflow was automated and implemented using a Python script. The classified binary map of impervious/non-impervious surface from the 1st level was used as the input. The PIC was assigned to the central pixel of the moving window. The output is a multi-layer image with three bands, one for each window size. To reduce the multi-layer image to a single-band product with $0.3 \mathrm{~m}$ spatial resolution we averaged the three bands with equal weights (Eq. 1):

$$
\text { Weightedaverage }=\frac{P I C_{100}+P I C_{400}+P I C_{800}}{3}
$$

The derived weighted average image was normalized to scale of 0 to 1 to retrieve the degree of urbanity. A high value corresponds to high urbanization and a low value to less urbanization based on the 2D metric alone. The map allows classifying any point into a level of urbanity based on its environment.

\subsection{Green and grey infrastructure complexity (3rd level)}

The third level combines 2D information with 3D information on buildings and tree or green volumes. The first step was to extract an accurate digital surface model (DSM) from WorldView-3 satellite stereo pairs. For this purpose, we used the OrthoEngine ${ }^{\mathrm{SE}}$ from Geomatica Banff ( $\mathrm{PCl}$ Geomatics, Richmond Hill, Ontario, Canada). A moving window of $100 \mathrm{~m} \times 100 \mathrm{~m}$ was applied over the DSM to extract the lowest $1 \%$ quantile representing the ground elevation within each square. A normalized height model (NHM) for each square was derived by subtracting the ground elevation value from the DSM. The space filling volume of buildings was estimated from the average of the NHM per building polygon, where the building polygon was extracted from the classified built-up area map. The volume of buildings was then calculated from the building polygon and its mean height. The tree volume was estimated accordingly and corresponds to that of a pillar above the crown projection area with tree height.

From the three indicator variables "mean impervious cover \%", "building volume per ha" and "tree volume per ha" we identified clusters with similar overall variable profiles regardless of their absolute magnitudes; this is a common grouping approach taken from gene expression data analysis. First, we computed the correlationbased distance using the function get_dist() [in factoextra R package], which uses the Pearson correlation to quantify the similarity of value profiles over the three ordered indicator variables. Second, a hierarchical clustering was applied from hclust) with $k=5$ clusters which was the optimal number of clusters identified using the gap statistics method fviz_nbclust() function [in factoextra R package].

\section{Results}

\subsection{Impervious surface, built-up, and tree cover mapping}


The results of the classification for the three classes "tree cover", "built-up", and "impervious surface" are shown in Fig. 2 for a subset of $1 \mathrm{~km} \times 1 \mathrm{~km}$. The image shows a good match with the underlayed WorldView-3 imagery. The overall accuracy (OA) is $89 \%$ for tree cover, $92 \%$ for impervious surface, and $87 \%$ for built-up respectively. We used an independent validation dataset of size $n=200$ points that have been classified based on visual interpretation. Although the classification outcomes were convincing, it is also visible in Figure 2 that the network partly failed to separate single buildings from each other.

\subsection{Categorization of green and grey infrastructure}

Figure 3 shows the averaged variable profiles of the separated five clusters. The variable Mean PIC, which contains the spatial aggregation over moving windows of different sizes, identified four separable clusters where each represents a different degree of urbanization. Cluster \#1 and \#2 are very close to each other and, in view of the profile, describe unsealed areas with vegetation or arable/ fallow land, etc. Table 1 gives a more detailed description of the cluster characteristics.

Cluster description

Illustrative image

Cluster \#1 is characterized by a very low percentage of impervious surface and a very low volume of buildings and trees. It describes a sparsely populated area with farmland or fallow land close to denser populated areas.

Cluster \#2 shows the largest amount of tree volume together with a low mean impervious cover \% in the surroundings. Combined with the non-existent building volume, this cluster embraces plantations, green spaces such as parks and agricultural land use.

Cluster \#3 is characterized by a high mean impervious cover \% in the surroundings and a high amount of building volume which indicate multi-floor buildings together with moderate tree volume sourced from roadside or sparsely distributed single trees.

Cluster \#4 shows the highest mean impervious cover \% in the surroundings and a moderate built-up volume per hectare. The tree volume for this type of area is quite low, thus, this cluster can be categorized as a highly-dense urban area.

Cluster \#5 is compromised by a moderate mean impervious cover \% in the surroundings, less built-up volume but compared to the previous cluster, cluster \#5 shows a higher tree volume.

\subsection{Mapping green and grey infrastructure complexity}


Our map of the complete study area (Fig. 4a) shows the spatial distribution of the five clusters from correlationbased distance clustering on a continuous scale. The red coloured area comprises built-up area, other types of impervious surfaces and a few green spaces. It also becomes clear that there is all but a linear urbanrural gradient within the research transect. Instead, the spatial distribution of the individual clusters shows that, although there is a clumping of cluster \#4 in the area close to the city center, this cluster is also found in more distant areas that may have similar characteristics with respect to the input variables.

Figure 4 (B). shows the spatial distribution of the villages in the Northern transect and their assignment to the respective clusters. A closer look into the map shows that on a small scale there are two regions in which cluster \#1 to \#4 occur together. Fifty percent of the villages were classified into cluster 2, while $20 \%$ each fell into clusters 3 and 4 . Only $10 \%$ of the villages were attributed to cluster 1 (Fig. 5).

\section{Discussion}

Keeping urbanization within sustainable limits, and harnessing green infrastructure such as urban trees for this endeavour, are big future challenges (Elmqvist and Maddox, 2018) against the background that it is expected that five billion people will live in an urban environment by 2030 (Venugopal et al., 2010). There is a need to collect meaningful information to support decision processes in urban planning, most notably regarding the future distribution of green infrastructures (Haaland and van den Bosch, 2015) .

With the aim of contributing to satisfy these information needs, the present study provides a data-driven approach that characterizes rural-urban gradients in terms of urbanity and grey/green infrastructure complexity. The produced map combines the percent impervious surface on a 2D scale and the presence of trees and buildings and their corresponding volumes on a 3D scale as indicators to quantify the degree of urbanity, with implications for urban quality.

Five clusters of similar characteristics were discriminated; each of these five clusters characterizes urban quality or quality of life in specific ways, and these contributions can be either positive or negative. Cluster \#4 is characterized by a high coverage of impervious surface and only minor tree volume, and is the dominant type in Urban Bengaluru. Relatively few trees in combination with a high coverage of impervious surface coverage may have negative effects on quality of life and a healthy urban community. In terms of air quality, McPherson et al. (1994) mentioned that particularly the large trees, like those huge alley trees in Bengaluru, remove up to 70 times more air pollution than small trees. Street trees play a key role for quality of life (Turner-Skoff and Cavender, 2019) and therefore, there should be minimum criteria for the amount of tree cover available. For example, van den Bosch (2021) recently proposed a 3-30-300m rule, according to which one should be able to see 3 trees from each house and the neighbourhood canopy coverage should be at least $30 \%$ to provide environmental, economic or social benefits (Mullaney et al., 2015) and lastly, there should be a green space at $300 \mathrm{~m}$ distance. 
Regions where neighbouring villages are categorized differently in terms of the grey and green infrastructure complexity may be an indicator for an ongoing transformation towards a more urban environment. The presented cluster approach can maybe a first indication for new type of environment that could be named "rurban". Rurban environments do not necessarily represent an intermediate state along a linear transition from urban to rural, but often lead to highly dynamic, complex and novel land cover constellations due to multiple tensions, development processes etc.

Tree cover is a very broad and general land cover class. Cluster \#2 in particular includes mango plantations as well as urban parks - although these different types of tree cover provide very different ecosystem services and thus also contribute to quality of life in very different ways (one point is the question of public access, which is not necessarily guaranteed in the case of plantations, for example). As a future extension, we plan to implement a more detailed classification scheme which may provide more linkages to the different ecosystem services and to quality of life.

The three input maps used in the clustering process may have some shortcomings, such as misclassification. Although the classification is based on very high-resolution imagery, mainly pure pixels per land cover class and a state-of-the-art classifier, it cannot be avoided that some objects or land cover types have been incorrectly classified. This in turn may have only a minor impact due to the coarser grid resolution but will nonetheless misallocate some clusters. The grid resolution of the presented continuous map of $100 \mathrm{~m} \times 100 \mathrm{~m}$ is in line with Schoepfer et al. (2005) who came up with a $100 \mathrm{~m}$ grid after an exchange with city planners. In comparison, the resolution of the maps used for the analysis is very high with $0.3 \mathrm{~m}$. However, for an area of $250 \mathrm{~km}^{2}$, the analysis is computationally intensive. Down sampling to 1 ha during cluster analysis is a compromise of computational requirements, preservation of spatial variability, and interpretability of the map. The down sampling also offers the possibility to upscale this approach by integrating Sentinel-2 satellite imagery and monitor changes in categorization over time.

\section{Conclusion}

Rapid urbanization creates new and complex configurations of land that are not captured by conventional dichotomies of "urban" and „rural" - in particular in the Global South. At the same time there is evidence of the important role of green infrastructure in mitigating the negative impacts of urbanization on sustainability. We make use of availability of 3D stereo satellite imagery and develop a data-driven approach to characterize a rural-urban interface regarding its green and grey infrastructure complexity. This is novel in terms of the method following an unsupervised and objective approach and the integration of 3D information. The method could be taken up in urban planning and in particular in green infrastructure development as it provides spatial information and a categorization of green infrastructure. For further development of the approach, an assessment of ecosystem services from green infrastructure would complement the information basis for decision making. Our study contributes to the existing recommendations from Hansen et al. (2019) for planning green infrastructure in cities and allows planning and site development by using a wall-to-wall map of green infrastructure categories. 
Further, our approach allows identifying and mapping typical land use or land system archetypes with similar variable profiles that can be correlated or enriched by socio-economic and ecological data to allow for further analysis of different social-ecological contexts(Rocha et al., 2020).

\section{Declarations}

\section{Funding}

The authors gratefully acknowledge the financial support provided by the German Research Foundation, DFG, through grant number 279374797 (Research Unit FOR2432/2).

\section{Competing Interests}

The authors have no relevant financial or non-financial interests to disclose.

\section{Availability of data and material}

Not applicable

\section{Code availability}

Not applicable

\section{Author Contributions}

All authors contributed to the study conception and design. Material preparation, data collection and analysis were performed by Nils Nölke. The first draft of the manuscript was written by Nils Nölke and all authors commented on previous versions of the manuscript. All authors read and approved the final manuscript.

\section{References}

1. Cablk ME, Minor TB (2003) Detecting and discriminating impervious cover with high-resolution IKONOS data using principal component analysis and morphological operators. Int J Remote Sens 24:4627-4645. https://doi.org/10.1080/0143116031000102539

2. Chollet F (2017) Deep Learning with Python. Simon and Schuster

3. Elmqvist T, Andersson E, McPhearson T, Bai X, Bettencourt L, Brondizio E, Colding J, Daily G, Folke C, Grimm N, Haase D, Ospina D, Parnell S, Polasky S, Seto KC, van der Leeuw S (2021) Urbanization in and for the Anthropocene. https://doi.org/10.1038/s42949-021-00018-w. npj Urban Sustain 1

4. Elmqvist T, Maddox D (2018) Urban planet: Knowledge towards sustainable cities. Cambridge University Press, Cambridge, p 482

5. Elvidge CD, Tuttle BT, Sutton PS, Baugh KE, Howard AT, Milesi C, Bhaduri BL, Nemani R (2007) Global distribution and density of constructed impervious surfaces. SENSORS 7:1962-1979 
6. European Commission. Statistical Office of the European Union (2021) Applying the degree of urbanisation: a methodological manual to define cities, towns and rural areas for international comparisons: 2021 edition. Publications Office, LU

7. Freudenberg M, Nölke N, Agostini A, Urban K, Wörgötter F, Kleinn C (2019) Large Scale Palm Tree Detection In High Resolution Satellite Images Using U-Net. Remote Sensing 11:312. https://doi.org/10.3390/rs11030312

8. Goetz SJ, Wright RK, Smith AJ, Zinecker E, Schaub E (2003) IKONOS imagery for resource management: Tree cover, impervious surfaces, and riparian buffer analyses in the mid-Atlantic region. Remote Sens Environ 88:195-208. https://doi.org/10.1016/j.rse.2003.07.010

9. Groenewegen PP (2006) Vitamin G: effects of green space on health, well-being, and social safety. BMC Public Health 6:1-9. https://doi.org/10.1186/1471-2458-6-149

10. Haaland C, van den Bosch CK (2015) Challenges and strategies for urban green-space planning in cities undergoing densification: A review. Urban Forestry \& Urban Greening 14:760-771. https://doi.org/10.1016/j.ufug.2015.07.009

11. Hansen R, Olafsson AS, van der Jagt AP, Rall E, Pauleit S (2019) Planning multifunctional green infrastructure for compact cities: What is the state of practice? Ecol Ind 96:99-110. https://doi.org/10.1016/j.ecolind.2017.09.042

12. Hiremath S, Prabhura DK, Lakshmikantha BP, Chakraborty SD (2013) Land Use/Land Cover Change Analysis of BangaloreUrban District and Its Impact on Land Surface Temperature

13. Hoffmann E, Jose M, Nölke N, Möckel T (2017) Construction and Use of a Simple Index of Urbanisation in the Rural-Urban Interface of Bangalore, India. Sustainability 9, 2146. https://doi.org/10.3390/su9112146

14. Hu X, Weng Q (2011) Impervious surface area extraction from IKONOS imagery using an objectbased fuzzy method. GEOCARTO INTERNATIONAL 26:3-20. https://doi.org/10.1080/10106049.2010.535616

15. Iglovikov V, Mushinskiy S, Osin V (2017) Satellite Imagery Feature Detection using Deep Convolutional Neural Network: A Kaggle Competition. http://arxiv.org/pdf/1706.06169v1

16. Kleinn $C$ (2001) A cautionary note on the minimum crown cover criterion in forest definitions. CANADIAN JOURNAL OF FOREST RESEARCH-REVUE CANADIENNE DE RECHERCHE FORESTIERE 31, 350-356

17. Lepczyk CA, Aronson MFJ, Evans KL, Goddard MA, Lerman SB, Maclvor JS (2017) Biodiversity in the City: Fundamental Questions for Understanding the Ecology of Urban Green Spaces for Biodiversity Conservation. BioScience 67, 799-807. https://doi.org/10.1093/biosci/bix079

18. Liu Z, He C, Zhou Y, Wu J (2014) How much of the world's land has been urbanized, really? A hierarchical framework for avoiding confusion. Landscape Ecol 29:763-771. https://doi.org/10.1007/s10980-014-0034-y

19. Ma Q (2016) A hierarchical analysis of the relationship between urban impervious surfaces and land surface temperatures: spatial scale dependence, temporal variations, and bioclimatic modulation. 
Landscape Ecol 1-15. https://doi.org/10.1007/s10980-016-0356-z

20. Maas J, Spreeuwenberg P, van Winsum-Westra M, Verheij RA, de Vries S, Groenewegen PP (2009) Is green space in the living environment associated with people's feelings of social safety? Environ. Plann A 41:1763-1777. https://doi.org/10.1068/a4196

21. Magdon P, Fischer C, Fuchs H, Kleinn C (2014) Translating criteria of international forest definitions into remote sensing image analysis. Remote Sens Environ 149:252-262.

https://doi.org/10.1016/j.rse.2014.03.033

22. Abadi M, Barham P, Chen J, Chen Z, Davis A, Dean J, Devin M, Ghemawat S, Irving G, Isard M, Kudlur M, Levenberg J, Monga R, Moore S, Murray DG Benoit Steiner, Paul Tucker, Vijay Vasudevan, Pete Warden, Martin Wicke, Yuan Yu, Xiaoqiang Zheng, 2005. TensorFlow: A System for Large-Scale Machine Learning, in: Papers presented at the 2005 workshop on Wireless traffic measurements and modeling. 12th \{USENIX\} Symposium on Operating Systems Design and Implementation (\{OSDI\} 16). USENIX Association, Berkeley, CA, pp. 265-283

23. Mullaney J, Lucke T, Trueman SJ (2015) A review of benefits and challenges in growing street trees in paved urban environments. Landscape and Urban Planning 134:157-166.

https://doi.org/10.1016/j.landurbplan.2014.10.013

24. Nagendra $\mathrm{H}$ (2016) Nature in the city: Bengaluru in the past, present, and future. Univ. Press, Oxford, p 224

25. Nowak DJ, Crane DE, Stevens JC (2006) Air pollution removal by urban trees and shrubs in the United States. Urban Forestry \& Urban Greening 4:115-123. https://doi.org/10.1016/j.ufug.2006.01.007

26. Rocha J, Malmborg K, Gordon L, Brauman K, DeClerck F (2020) Mapping social-ecological systems archetypes. ENVIRONMENTAL RESEARCH LETTERS 15. https://doi.org/10.1088/1748-9326/ab666e

27. Ronneberger O, Fischer P, Brox T (2015) U-Net: Convolutional Networks for Biomedical Image Segmentation, in: Medical image computing and computer-assisted intervention - MICCAI 2015: 18th international conference, Munich, Germany, October 5-9, 2015: proceedings, part III, Cham. 2015. Springer, Cham, Heidelberg, New York, Dordrecht, London, pp. 234-241

28. Schoepfer E, Lang S, Blaschke T (2005) A “ Green Index ". Incorporating Remote Sensing and Citizen " $S$ Perception of Green Space

29. Seto KC, Fragkias M, Güneralp B, Reilly MK (2011) A meta-analysis of global urban land expansion. PLoS ONE 6:e23777. https://doi.org/10.1371/journal.pone.0023777

30. Sudhira HS, Nagendra H (2013) Local Assessment of Bangalore: Graying and Greening in Bangalore - Impacts of Urbanization on Ecosystems, Ecosystem Services and Biodiversity., in: In: Elmqvist T, Fragkias M, Goodness J, Güneralp B, Marcotullio PJ, McDonald RI, Parnell S, Schewenius M, Sendstad M, Seto KC, Wilkinson C (eds) Urbanization, Biodiversity and Ecosystem Services: Challenges and Opportunities. Springer Netherlands, pp 75-91

31. Troy A, Grove JM (2008) Property values, parks, and crime: A hedonic analysis in Baltimore, MD. Landscape and Urban Planning 87:233-245. https://doi.org/10.1016/j.landurbplan.2008.06.005 
32. Turner-Skoff JB, Cavender N (2019) The benefits of trees for livable and sustainable communities. Plants People Planet 1:323-335. https://doi.org/10.1002/ppp3.39

33. van den Bosch CK (2021) Promoting health and wellbeing through urban forests - Introducing the 330-300 rule | Linkedln. https://www.linkedin.com/pulse/promoting-health-wellbeing-through-urbanforests-rule-cecil/?trackingld= (accessed 16 July 2021)

34. Venugopal RK, Ramesh B, Bhavani SVL, Kamini J (2010) Urban and regional planning, in: Roy, P.S., Dwivedi, R.S., Vijayan, D. (Eds.), Remote sensing applications. Hyderabad

35. Weng Q (2012) Remote sensing of impervious surfaces in the urban areas: Requirements, methods, and trends. Remote Sensing of Urban Environments 117:34-49.

https://doi.org/10.1016/j.rse.2011.02.030

\section{Figures}

\section{Figure 1}

Location of the study area, a transect of $50 \mathrm{~km} \times 5 \mathrm{~km}$ in the Northern part of Bengaluru, India. The transect is enlarged here as a WordView-3 false colour composite. 


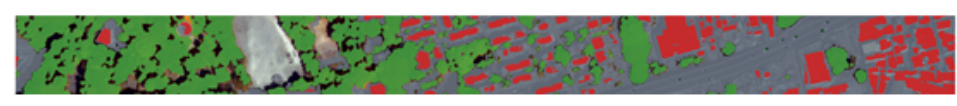

\section{Figure 2}

$1000 \mathrm{~m} \times 1000 \mathrm{~m}$ subset in urban Bengaluru shows the result of U-Net segmentation for three classes tree cover, built-up and impervious surface where built-up area is a sub-group of imperviousness. 


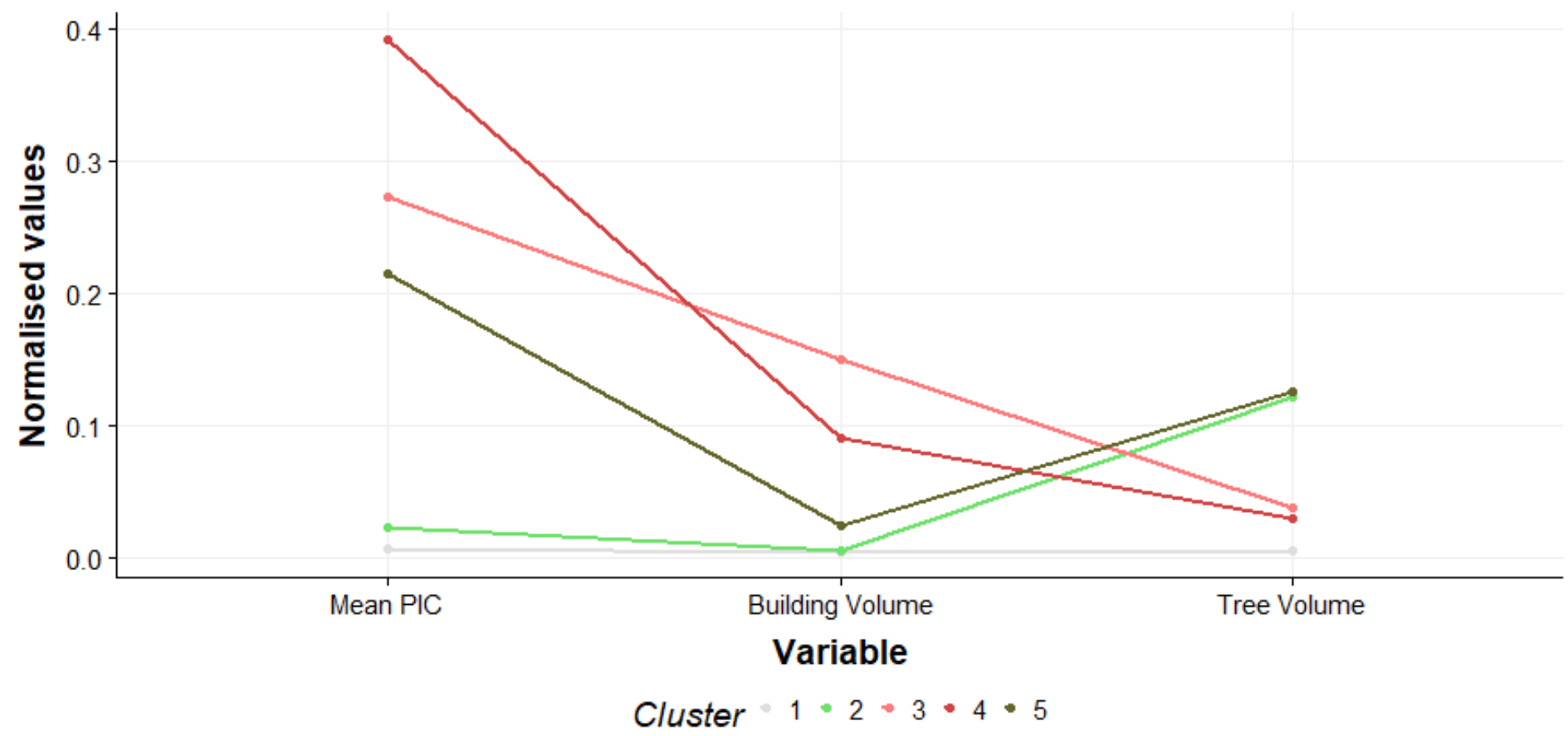

Figure 3

Different profile curves of the five clusters of the 3 variables (Mean PIC [2D], Building volume [3D] and Tree volume [3D])

\section{Figure 4}

Wall-to-wall mapping of the five clusters (a), and classification of the 98 villages showing a mix of clusters even far outside of the city centre (b) in the Northern transect. 
A

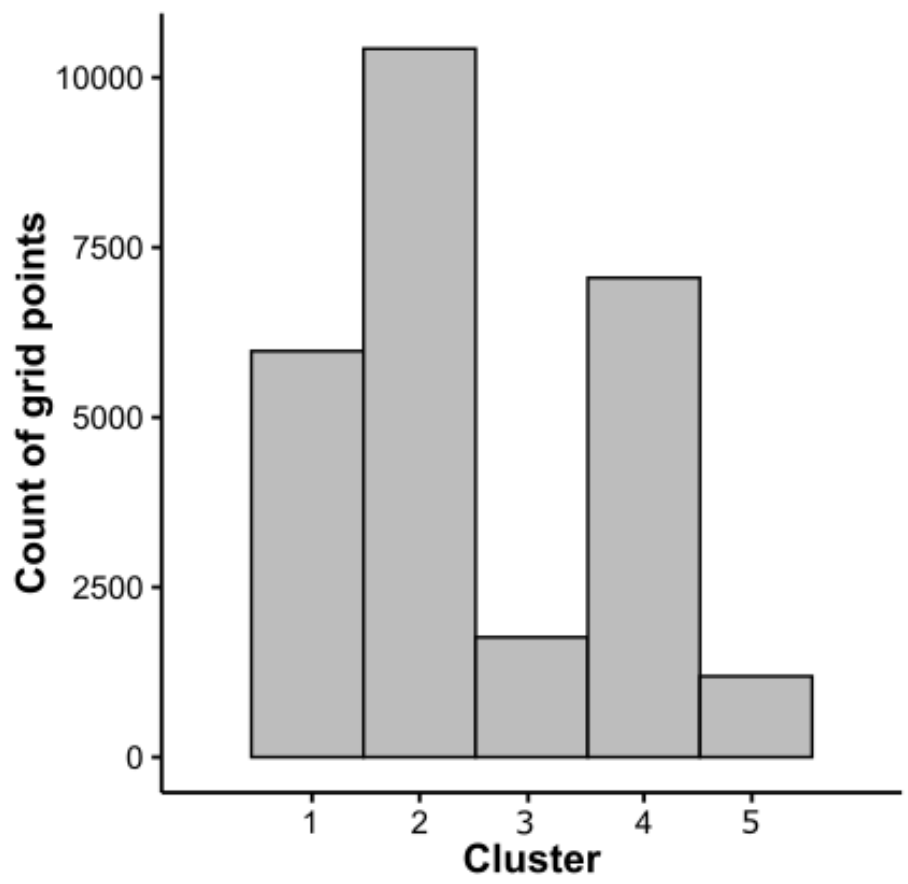

B

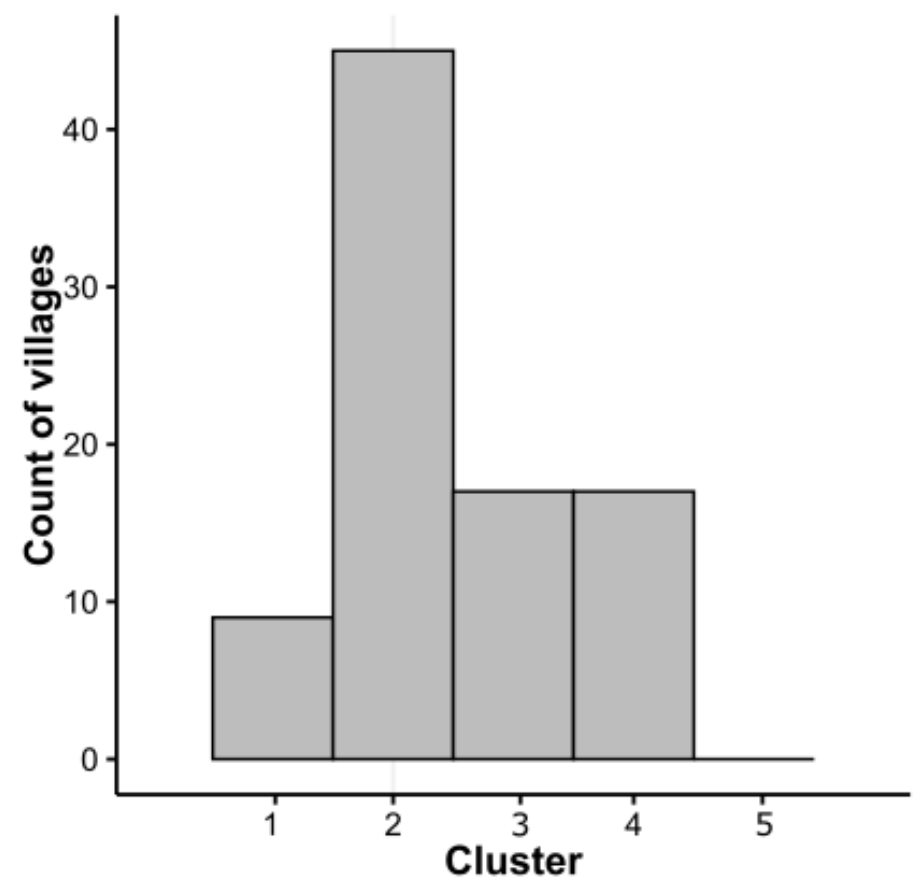

Figure 5

Frequency distribution of (a) villages and (b) the $100 \mathrm{~m} \times 100 \mathrm{~m}$ grid points within the transect for the five clusters. 